YAP, P. M. (1965a) Koro-a culture-bound deperson alization syndrome. British Journal of Psychiatry, 111, 43-50.

- (1965b) Koro in a Briton. British Journal of Psychiatry, $111,774-5$.

\section{L-TRYPTOPHAN IN MATERNITY BLUES}

DeAR SiR,

We were most interested to read Dr Harris' report (Journal, September 1980, 137, 233-35) of a doubleblind trial of L-tryptophan in the puerperium. The failure of exogenous L-tryptophan to affect the incidence of severity of maternity blues is probably not surprising. In a recent study (Journal, May 1980, $136,498)$ we showed that whether or not the blues is associated with lowered free tryptophan is strongly affected by seasonal factors. In addition, we showed that, in our subjects, failure of total tryptophan to rise after parturition was a significant indicator not only of the blues but of complaints of depression in the ensuing six months.

It is difficult to see how such a brief disturbance of tryptophan at parturition could bear a causal relationship to outcome at six months and accordingly we suggested that it may indicate an occult disturbance in tryptophan handling, perhaps related to a more generalized membrane transport disorder, which may be a predisposing factor for the development of depression. Thus we envisaged that disturbances in tryptophan dynamics during the puerperium could be biological markers of susceptibility to depression rather than primary causative factors. Dr Harris' findings support this view and in this sense disturbances in total or free tryptophan at parturition may indeed be epiphenomena as he suggests, not of the blues, but of a more fundamental disturbance which does bear a causal relationship to depressed mood.

\section{Department of Pharmacy, University of Aston, Birmingham} Sheila HaNDley

Department of Psychiatry, London Hospital Medical College, London El

\section{THE ART OF MEASURING SEROTONIN} UPTAKE IN PLATELETS

\section{Dear Sir,}

Arora and Meltzer (Journal, October 1980, 137, 396-99) criticize the paper by Coppen et al (Journal, March 1980, 136, 235-38), mainly on methodological grounds. Their main point seems to be that when 5-HT uptake is determined at concentrations greater than $1 \mu \mathrm{M}$ of $5-\mathrm{HT}$, the effects of passive diffusion cannot be entirely corrected. According to my studies (and those of others) this is not correctprovided one uses an adequate 'blind value' to be subtracted from the measured uptake values (Lingjaerde, 1977). The easiest way to obtain this blind value is that used by Coppen et al: to add varying concentrations of 5-HT to samples kept in the cold. When this blind value is used, I find that the initial uptake rate at $37^{\circ} \mathrm{C}$ shows all the characteristics of saturable, active uptake, at least up to $8 \mu \mathrm{M}$ of $5-\mathrm{HT}$. Thus, it is completely blocked by low concentrations of antidepressants like clomipramine, and by omitting sodium or chloride from the incubation medium.

It may be possible to obtain the same data also by extrapolating back from the linear part of the uptake vs. concentration curve, as recommended by Stahl and Meltzer (1978), Tuomisto and Tukiainen (1976) and Tuomisto et al (1979). However, I find this method less satisfactory, because of the uncertainty in assessment of the 'linear part' of the (hyperbolic) uptake curve.

Like Arora and Meltzer, I was surprised by Coppen et al's finding of reduced 5-HT uptake in plasma after adding lithium carbonate. In my own in vitro studies with lithium (added as chloride) I have never seen an inhibitory effect of lithium, even in high concentrations. Neither have I seen a stimulatory effect, except in the absence of $\mathrm{K}^{+}$; lithium thus seems to have a 'potassium-like' effect on 5-HT uptake (Lingjaerde, 1977). However, 5-HT uptake decreases very rapidly with increasing $\mathrm{pH}$ (Lingjaerde, 1977). Could it be that the inhibitory effect of lithium carbonate found by Coppen et al is due simply to increased $\mathrm{pH}$ ?

Indeed, the measurement of 5-HT uptake in platelets is blessed with many pitfalls!

Section of Clinical Psychiatry, ODD LINGJAERDE

University of Tromso, 9010 Asgard, Norway

\section{References}

LiNGJAERDE, O. (1977) Platelet uptake and storage of serotonin. In Serotonin in Health and Disease, Vol. 4 (ed. W. B. Essman). New York: Spectrum Publications.

StAhl, S. M. \& Meltzer, H. Y. (1978) A kinetic and pharmacologic analysis of 5-hydroxytryptamine transport by human platelets and platelet storage granules: comparison with central serotonergic neurons. Journal of Pharmacology and Experimental Therapeutics, 205, 118-32.

TUOMISTo, J. \& TUKIAINEN, E. (1976) Decreased uptake of 5-hydroxytryptamine in blood platelets from depressed patients. Nature, 262, 596-8.

\& AHLFORS, U. G. (1979) Decreased uptake of 5-hydroxytryptamine in blood platelets from patients with endogenous depression. Psychopharmacology, $65,141-7$. 